\title{
EXTREMAL HOLOMORPHIC IMBEDDINGS BETWEEN THE BALL AND POLYDISC ${ }^{1}$
}

\section{H. ALEXANDER}

\begin{abstract}
The following problem of Fornaess and Stout is considered: Find, among all polydiscs imbedded in the unit ball, the one which contains the largest ball centered at the origin.
\end{abstract}

Fornaess and Stout [1] have observed (as a consequence of their result that a monotone union of (imbedded) polydiscs in a taut complex manifold is (biholomorphic to) a polydisc) that an imbedded polydisc in the unit ball in $\mathbf{C}^{m}(m>1)$ cannot contain balls centered at the origin of radius arbitrarily close to one. More precisely, there is a positive number $R_{0}(m)<1$ such that the image of the unit polydisc $U^{m}$ by an imbedding in the unit ball $B_{m}$ in $\mathbf{C}^{m}$ never contains $s B_{m}$ for $s>R_{0}(m)$, where, for $W \subseteq \mathbf{C}^{m}, s W=\{s z: z \in W\}$. Nevertheless, $B_{m}$ contains an imbedded polydisc of full measure [1]. Fornaess and Stout, by exhibiting the map $F_{0}: U^{m} \rightarrow B_{m}, F_{0}(z)=z / \sqrt{m}$ with $F_{0}\left(U^{m}\right)$ $\supseteq B_{m} / \sqrt{m}$, showed that $R_{0}(m) \geqslant 1 / \sqrt{m}$ and raised the problem of finding the smallest (or any specific) value of $R_{0}(m)$. We show that, in fact, the smallest value of $R_{0}(m)$ is $1 / \sqrt{m}$ and that $F_{0}$ is the unique extremal imbedding, up to automorphisms.

PROPOSITION 1. If $F: U^{m} \rightarrow B_{m}$ is a holomorphic imbedding for which $F\left(U^{m}\right) \supseteq s B_{m}$, then $s \leqslant 1 / \sqrt{m}$. Moreover, the equality $s=1 / \sqrt{m}$ holds if and only if $F=B \circ F_{0} \circ A$ where $A$ is a biholomorphism of $U^{m}$ and $B$ is a unitary transformation.

COROLlaRY 1. The polydisc $U^{m} / \sqrt{m} \subseteq B_{m}$ is maximal among imbedded polydiscs in $B_{m}$.

The reasoning of Fornaess and Stout also shows that there is a positive number $S_{0}(m)<1$ such that if $B_{m}$ is imbedded into $U^{m}$, then the image does not contain $s U^{m}$ for $s>S_{0}(m)$. Now consider the inclusion map $G_{0}: B_{m} \rightarrow$ $U^{m}, G_{0}(z)=z$. Then $G_{0}\left(B_{m}\right) \supseteq U^{m} / \sqrt{m}$ and so $S_{0}(m) \geqslant 1 / \sqrt{m}$. This is the extremal case:

Proposition 2. If $G: B_{m} \rightarrow U^{m}$ is a holomorphic imbedding such that $G\left(B_{m}\right) \supseteq s U^{m}$, then $s \leqslant 1 / \sqrt{m}$. Moreover the equality $s=1 / \sqrt{m}$ holds if and only if $G=G_{0} \circ B(\equiv B)$ where $B$ is a biholomorphism of $B_{m}$.

Received by the editors June 20, 1977.

AMS (MOS) subject classifications (1970). Primary 32H99.

'Research supported in part by NSF 7607287 A01. 
COROLLARY 2. The ball $B_{m} \subseteq U^{m}$ is maximal among imbedded dalls in the polydisc $U^{m}$.

Proof of Proposition 1. Let $\|z\|$ be the Euclidean norm for $z \in \mathbf{C}^{m}$. Assume, first, that $F(0)=0$. Write $F$ in a vector Taylor series: $F(z)=$ $\sum a_{\alpha} z^{\alpha}$, where $a_{\alpha} \in \mathbf{C}^{m}$. The almost everywhere defined boundary values of the bounded holomorphic function $F$ will also be denoted by $F$. Since lim inf $\left\|F\left(r e^{i \theta}, 0, \ldots, 0\right)\right\| \geqslant s$ as $r \uparrow 1$, we have

$$
s^{2} \leqslant \frac{1}{2 \pi} \int_{0}^{2 \pi}\left\|F\left(e^{i \theta}, 0,0\right)\right\|^{2} d \theta=\sum\left\{\left\|a_{\alpha}\right\|^{2}: \alpha \in \mathcal{S}_{1}\right\}
$$

where $\delta_{k}=\left\{\alpha=\left(\alpha_{1}, \ldots, \alpha_{m}\right): \alpha_{k}>0, \alpha_{j}=0\right.$ for $\left.j \neq k\right\}$ for $k=$ $1,2, \ldots, m$. In the same way we get $s^{2} \leqslant \Sigma\left\{\left\|a_{\alpha}\right\|^{2}: \alpha \in \mathcal{S}_{k}\right\}$. Adding these $m$ inequalities, we have

$$
\begin{aligned}
m s^{2} & \leqslant \sum\left\{\left\|a_{\alpha}\right\|^{2}: \alpha \in \delta_{1} \cup \delta_{2} \cup \cdots \delta_{m}\right\} \\
& \leqslant \sum\left\|a_{\alpha}\right\|^{2} \leqslant \int_{T^{m}}\|F\|^{2} d h \leqslant 1,
\end{aligned}
$$

where $h$ is Haar measure on the torus $T^{m}$; we are using $a_{\alpha}=0$ for $\alpha=0$ and $\|F\|^{2} \leqslant 1$ a.e. on $T^{m}$. Thus $s \leqslant 1 / \sqrt{m}$.

In the case of equality $s=1 / \sqrt{m}$ we have (i) $a_{\alpha}=0$ for $\alpha \notin \delta_{1} \cup \delta_{2}$ $\cup \cdots \cup \delta_{m}$, (ii) $\left\|F\left(0, \ldots, e^{i \theta}, \ldots, 0\right)\right\|=1 / \sqrt{m}$ a.e. on the unit circle, where $e^{i \theta}$ is in the $k$ th position, for $1 \leqslant k \leqslant m$, and (iii) $\|F\|=1$ a.e. on $T^{m}$. By (i) and (ii) we can write $F\left(z_{1}, z_{2}, \ldots, z_{m}\right)=F_{1}\left(z_{1}\right)+F_{2}\left(z_{2}\right)+\cdots+$ $F_{m}\left(z_{m}\right)$ for $F_{k}: U \rightarrow \mathbf{C}^{m}$ with $F_{k}(0)=0$ and $\left\|F_{k}\left(e^{i \theta}\right)\right\|=1 / \sqrt{m}$ a.e. on the circle; abusing notation, we shall also view $F_{k}$ as a mapping defined on $U^{m}$ which depends only on the $k$ th variable.

For $z, w \in \mathbf{C}^{m}$ put $\langle z, w\rangle=\Sigma z_{j} \bar{w}_{j}$, the standard Hermitian inner product. Then $\operatorname{Re}\langle z, w\rangle$ is the standard real inner product on $R^{2 m}=\mathbf{C}^{m}$. Now (ii) and (iii) imply for almost all $p=\left(e^{i \theta_{1}}, \ldots, e^{i \theta_{m}}\right) \in T^{m}$,

$$
\begin{aligned}
1 & =\|F(p)\|^{2}=\sum\left\langle F_{k}\left(e^{i \theta_{k}}\right), F_{j}\left(e^{i \theta_{j}}\right)\right\rangle \\
& =\sum_{1}^{m}\left\|F_{k}(p)\right\|^{2}+2 \operatorname{Re} \sum_{k<j}\left\langle F_{k}\left(e^{i \theta_{k}}\right), F_{j}\left(e^{i \theta_{j}}\right)\right\rangle \\
& =\sum_{1}^{m} \frac{1}{m}+2 Q\left(e^{i \theta_{1}}, e^{i \theta_{2}}, \ldots, e^{i \theta_{m}}\right)
\end{aligned}
$$

where $Q\left(z_{1}, \ldots, z_{m}\right)=\operatorname{Re} \Sigma_{k<j}\left\langle F_{k}\left(z_{k}\right), F_{j}\left(z_{j}\right)\right\rangle$. Thus $Q=0$ a.e. on $T^{m}$. As $Q$ is a bounded $m$-harmonic function on $U^{m}$, it follows (see [2]) that $Q \equiv 0$ on $U^{m}$. Thus $\operatorname{Re}\left\langle F_{k}\left(z_{k}\right), F_{j}\left(z_{j}\right)\right\rangle=Q\left(0, \ldots, z_{k}, \ldots, z_{j}, \ldots, 0\right)=0$ for $\left|z_{j}\right|$ $<1,\left|z_{k}\right|<1$; i.e., $F_{k}\left(z_{k}\right)$ is real orthogonal to $F_{j}\left(z_{j}\right)$. Define $H_{j}$ to be the real linear span in $\mathbf{C}^{m}$ of the set $F_{j}(U)$. We have proved that the $H_{j}$ are mutually (real) orthogonal. Since $F$ is one-to-one, it follows that each $F_{j}$ is one-to-one on $U$. Therefore, $\operatorname{dim}_{\mathbf{R}} H_{j} \geqslant 2$. We conclude that $\operatorname{dim}_{\mathbf{R}} H_{j}=2$ for each $j$ and that $H_{j}$ is complex linear (a "complex line"). Now, replacing $F$ by $V \circ F$ 
where $V$ is a unitary transformation taking $H_{j}$ to the $j$ th complex coordinate axis, we may assume that $H_{j}$ is the $j$ th coordinate axis. Thus $F_{j}\left(z_{j}\right)=$ $\left(0, \ldots, f_{j}\left(z_{j}\right), \ldots, 0\right)$ where, in the $j$ th position, there is a bounded complex valued holomorphic function $f_{j}$ defined on $U$ and satisfying $f_{j}(0)=0$ and $\left|f_{j}\left(e^{i \theta}\right)\right|=1 / \sqrt{m}$ a.e.. Thus $\sqrt{m} f_{j}$ is an inner function. Since $f_{j}$ is one-toone we conclude that $f_{j}(\zeta)=e^{i \beta j \zeta / \sqrt{m}}$ for real $\beta_{j}$. This proves that $F=$ $B \circ F_{0}$ where $B$ is a unitary transformation.

We have assumed that $F(0)=0$. The general case can be reduced to this case by preceding $F$ by an automorphism of $U^{m}$.

Proof of Corollary 1. If $P$ is an imbedded polydisc in $B_{m}$ containing $U^{m} / \sqrt{m}$, then $P$ contains $B_{m} / \sqrt{m}$ and by Proposition 1, there is a unitary transformation $B$ such that $P=B\left(U^{m} / \sqrt{m}\right)$. It follows that $B\left(T^{m} / \sqrt{m}\right)=T^{m} / \sqrt{m}$ and hence $\left|z_{k}\right| \leqslant 1 / \sqrt{m}$ on $P$. Therefore $P=U^{m} / \sqrt{m}$.

Proof of Proposition 2. By preceding $G$ by an automorphism of $B_{m}$, we may assume, without loss of generality, that $G(0)=0$. Let $\varepsilon>0$. Let $F=$ $\left(G^{-1} \mid s U^{m}\right): s U^{m} \rightarrow B_{m}$. Applying Proposition 1 to $F$, we see that $F\left(s U^{m}\right)$ does not contain $(1+\varepsilon) / \sqrt{m} B_{m}$. Thus there is a point $p \in B_{m} \backslash F\left(s U^{m}\right)$ with $\|p\|<(1+\varepsilon) / \sqrt{m}$. Hence $q \equiv G(p) \notin s U^{m}$ and so one of the coordinates, say $q_{k}$, of $q$ satisfies $\left|q_{k}\right| \geqslant s$. Schwarz's lemma applied to the $k$ th component $g_{k}$ of $G$ yields $\left|g_{k}(z)\right| \leqslant\|z\|$ for all $z \in B_{m}$. Thus $s<\left|q_{k}\right|=\left|g_{k}(p)\right| \leqslant\|p\|<(1+\varepsilon) / \sqrt{m}$; i.e., $s \leqslant 1 / \sqrt{m}$.

In order that $s=1 / \sqrt{m}, F\left(U^{m} / \sqrt{m}\right)$ must contain $B_{m} / \sqrt{m}$. From Proposition 1 we conclude that $F$ is a unitary transformation (restricted to $U^{m} / \sqrt{m}$ ). This implies that $G$ has the desired form.

Corollary 2 follows directly from Proposition 2.

\section{REFERENCES}

1. J. E. Fornaess and E. L. Stout, Polydiscs in complex manifolds, Math. Ann. 227 (1977), 145-153.

2. Walter Rudin, Function theory in polydiscs, Benjamin, New York, 1969.

Department of Mathematics, University of Illinois at Chicago Circle, Chicago, ILLINOIS 60680 